\title{
COMMENTARY
}

\section{When the Simulation Becomes Real}

\author{
Allison M Dering-Anderson, PharmD, ${ }^{\mathrm{a}}$ Michael A Mone, JD, ${ }^{\mathrm{b}}$ Paul P Dobesh, PharmD ${ }^{\mathrm{a}}$ \\ ${ }^{a}$ University of Nebraska, College of Pharmacy, Omaha, Nebraska \\ ${ }^{\mathrm{b}}$ CVS Health
}

Corresponding Author: Allison M Dering-Anderson, University of Nebraska, College of Pharmacy, 986145 Medical Center, Omaha, NE 68198-6145. Tel: 402-432-2080. Email: Ally.DeringAnderson@UNMC.edu

Submitted January 23, 2021; accepted June 16, 2021; ePublished July 2021

Keywords: virtual simulated assessment, assessor, unexpected medical condition, planning simulation exercises

What should the evaluator, in a simulated learning experience, do when the assessment being scored yields a critical result? We searched for guidance on recommendations or standards for an evaluator becoming a provider and we were unable to find any published work addressing our experiences. There are numerous articles addressing standardized patients. ${ }^{1,2,34}$ An article by Simon and Dove ${ }^{5}$ encourages a professional evaluation of the standardized patient before assessment by learners. Thus, preventing the finding of urgent or emergent results during the skills assessment. Neither of our experiences used standardized patients. One used classmate-partners and the other a family volunteer to serve as the patients. We offer the following two experiences to initiate a discussion centering on serving as an evaluator in skills testing and the added challenge of serving as a remote evaluator for learners in other jurisdictions. Each of these experiences is based on real evaluator interactions but have been modified to protect the identity of the learners.

The first assessment was an in-person assessment of basic patient physical assessment skills as a part of a national point-of-care testing certificate training program. Both evaluators were pharmacists, licensed in the jurisdiction of the testing, with assistance from trained student-assessors who had previously achieved the national certificate. During blood pressure assessment, a pharmacy student learner with an unusually high reading $(182 / 94 \mathrm{mmHg})$ was identified. ${ }^{6}$ The student-assessor requested help from the pharmacist-evaluator. While taking the patient history, the pharmacist-evaluator identified previous treatment for rhabdomyolysis. ${ }^{7,8}$ The student was transported to the emergency department and was subsequently admitted for renal failure. The pharmacist-evaluator accompanied the student to the emergency department and stayed until a family member arrived.

The second assessment was a synchronous, online assessment of a diabetic foot examination. The learner was a pharmacist, licensed in the jurisdiction where the testing was being performed, the evaluator, also a pharmacist, was licensed in a different jurisdiction than where the state where the assessment was conducted. The learner was performing a micro-filament test and the simulated patient, a family member, was unable to feel any of the attempts at stimulation with the micro-filament. The simulated patient had no history of diabetes, leg, or spinal injury, and was able to feel digital stimulation when the learner used a finger to create sensation. ${ }^{9}$ The pharmacist-evaluator recommended a consultation with the patient's provider at the earliest possible opportunity, this advice was confirmed by the pharmacist-learner.

Evaluators do not expect to find problems during simulated skills assessments. Generally, the simulated patients are either healthy students, faculty, staff or standardized patients with known conditions. In these two instances, however, the simulated patient immediately became a real patient. The pharmacist-evaluators likewise immediately became providers. This challenge and the proper handling of these situations does not appear in the literature. We propose to start the discussion but do not claim to have all the answers.

\section{Assessment Design and Evaluator Considerations}

The identification of unexpected health issues is a part of the profession of pharmacy. Pharmacists are trained to manage these issues through team-based care and proper patient referral. As such, both of these examples provide an opportunity to highlight the clinical services pharmacists provide.

The two examples provided in this commentary, however, are not simulated, they are real. The people involved as simulation patients were identified to have medical needs; one an immediate need and the other a need for evaluation with limited delay. These experiences illuminate that the evaluator must be able to do more than properly complete a scoring rubric. The evaluator must also be able to appropriately manage problems identified during the simulated experience. We would propose that in preparing a simulated experience, the planning should include: crafting the experience thoughtfully 
to gather the information required for assessment; creating an appropriate scoring rubric; identifying any potential medical issues that could be identified during the simulation; and the selection of evaluators who are able to manage these identified medical issues should they arise during the exercise. For instance: if taking blood pressure, each evaluator should know what to do in the event that hypotension or hypertension are identified; when testing for sensation using a Semmes-Weinstein Monofilament Examination (SWME), the evaluator should be provided with proper action if no sensation is detected; when screening blood sugar, evaluators should be prepared with information regarding those levels that are cause for referral. Performing health assessment tests is, to some degree, within the scope of practice for pharmacists. We are not advocating that only a cardiology expert can assess blood pressure monitoring or that endocrine specialists are the only providers qualified to assess proper blood sugar testing, but we are encouraging preparation of evaluators to deal with abnormal findings. In those simulated laboratories where a critical or actionable result could be discovered, we are advocating that pharmacists or other appropriate health care providers be used as the evaluators. ${ }^{10}$

Not all clinical skills laboratories have the potential to identify underlying medical issues. A skills laboratory focusing on patient counseling, for instance, would not expect to discover problems because the simulated patient will be presenting from a deliberate, planned dialogue. ${ }^{11,12,13}$ Careful consideration should be given to collecting data during the development phase of a laboratory exercise. If the skill being assessed is a finger stick process, is it necessary to get a resulting blood sugar that may need to be acted upon? Or is the skill being assessed merely the collection of the blood sample? In some cases, it may be appropriate to remove the data gathering element in its entirety. This will prevent any conversion from simulation to "real" and will also protect health information that is specific to the simulated patient.

\section{Evaluator Ethical Response}

Preparing the evaluator for problem issues that may be identified during the simulated assessment is the responsibility of the professional developing the assessment activity. Planning how to respond, should these issues arise, is the responsibility of the evaluator. ${ }^{14,15,16}$ In fact, we would argue that responding appropriately is an ethical mandate for evaluators who are also health care professionals. Health care professionals, in our example pharmacists, are responsible for their actions when interacting with patients. As soon as the learner identifies a potential medical problem, the simulated patient becomes a real patient, and the pharmacist-evaluator becomes a provider. At that point, professional ethics must be considered. The evaluator is no longer serving the learner, the evaluator becomes the pharmacist provider serving the patient, with beneficence, nonmaleficence, fidelity, and veracity. ${ }^{17}$ Any professional asked to serve as an evaluator must determine, in advance, if they are prepared to meet this responsibility. If not, the request to be an evaluator should be declined.

\section{Evaluator Legal Response}

Is the pharmacist who agrees to serve as an evaluator "practicing pharmacy"? In most jurisdictions the practice of pharmacy does not extend to pharmacy education as a licensure obligated function. That is, faculty members are not required, as a matter of pharmacy law, to be licensed within the jurisdiction where they teach. Those engaging in clinical practice are licensed in order to provide patient care, but educators without a practice site are not required to be licensed, unless such requirements are imposed by the institution where they teach. What happens then when the health care professional evaluator is thrust into the position of provider by the discovery of a medical condition during the assessment?

In our first example, the pharmacist was licensed in the jurisdiction where the assessment was provided. That pharmacist could easily transition into the role of provider. In the second example, the pharmacist-evaluator was not licensed in the jurisdiction where the identified patient was located. This pharmacist-evaluator had no legal authority to practice the profession of pharmacy in that state, even though he was legally licensed in the jurisdiction where he was located. He was observing and interacting with the pharmacist-learner and the patient using online, video technology. In this capacity the evaluator was "in charge" of the learning experience and the findings.

Did he act professionally? A part of the answer may lie in the definition of telepharmacy in the state where the patient was located. It will also depend on the jurisdiction's definition of patient counseling. Certainly, each state has the duty to protect its citizens from unlicensed practice. But these unlicensed practice laws likely do not address this situation directly and the dearth of literature addressing the situation implies that regulators, like educators, have not considered these situations. Is it reasonable to try to demarcate the line between "advice" and "counseling"? Is it reasonable to require state licensure to assess skills in pharmacy education? No, neither are reasonable nor easily accomplished. While states have the responsibility to protect their citizens from unlicensed practice, they do not have a need to protect patients from receiving advice. As long as the patient can clearly determine whether the advice is being provided by a licensed health care provider or not, patients or caregivers can be allowed to make decisions based on advice. Additionally, the learner in this scenario was a pharmacist, licensed in the jurisdiction where the simulated assessment was being conducted. The 
learner-pharmacist also had a responsibility to the patient to provide appropriate care and advice. Colleges of Pharmacy cannot reasonably expect faculty to be licensed in 20 or 30 states to perform skills assessments, but they may be teaching students located in that many or more states and jurisdictions due to on-line activities.

\section{Evaluator and Sponsor Follow-Up}

The final considerations from these two encounters focus on the pharmacist's duty to follow-up on the patient's condition. In the first example, the pharmacist-evaluator, not the learner, gathered the patient history that identified the previous rhabdomyolysis. The pharmacist was on-site and personally evaluated the patient's blood pressure. This pharmacist accompanied the patient to the hospital and assured that the patient was properly referred to another member of the health care team. This pharmacist completed her duty to the patient. The patient was accurately assessed and delivered to proper care.

In the second encounter, the pharmacist-evaluator was at a distance, with no opportunity to personally conduct a foot sensation test using micro-filament. This pharmacist had to rely on the comments from the learner and the patient to understand that the patient lacked sensation. The pharmacist did not accompany the patient to another member of the health care team, rather, the pharmacist recommended contacting a primary care provider or podiatrist. With limited knowledge of the area where the patient lived, the pharmacist did not make a specific recommendation of which provider to see, only general recommendations. Both the patient and the pharmacist-learner said that they understood the recommendation and would seek medical help. The pharmacist-evaluator reported the circumstances to the faculty member responsible for the assessment activity.

Pharmacists, like any other member of the health care team, are responsible to assure that a referred patient has accessed the referral provider or to determine that the patient has chosen to ignore the advice. Failure to follow-up could constitute unprofessional conduct based on a breach of the applicable standard of care. The pharmacists described in these assessment encounters each followed through for the patient and for the faculty responsible for the educational session. Each of the pharmacists described here accepted the responsibility to become a provider when faced with information that a simulation patient had an identified medical need. Each of the pharmacists made a proper referral and each notified the person in charge of the situation. ${ }^{6,7,8,9}$ It could be argued that discussing these situations is not necessary as no adverse outcomes were noted and each professional acted professionally and ethically, in the best interest of the patient. We would argue that an $N$ of two is insufficient to demonstrate that this is the standard response. Further, we would argue that standards, determined by the educators and professionals designing and assessing simulated patient encounters are essential to assuring that the educational process demonstrates the highest quality of patient care and that the health care professionals involved in assessing simulated encounters have the guidance needed to be comfortable in these situations.

\section{REFERENCES}

1. Hassett JM, Zinnerstrom K, Nawotniak RH, et.al; Utilization of standardized patients to evaluate clinical and interpersonal skills of surgical residents; Surgery 2006; 140(4) 633-639. doi: 10.1016/j.surg.2006.07.014

2. Kao SP, Chen TY, Hsieh ML; Effectiveness of a training program on the role recognition and case portrayal of standardized patients ; Tzu Chi Med J 2018; 30(1) 37-40. doi: 10.4103/tcmj.tcmj 1318

3. Paramasivan A, Khoo D; Standardized Patients Versus Peer Role Play-Exploring the Experience, Efficacy, and Cost-Effectiveness in Residency Training Module for Breaking Bad News; J Surg Edu 2020; 77(2) 479-484. doi: 10.1016/j.jsurg.2019.10.009

4. Brender E, Burke A, Glass R; Standardized Patients; JAMA 2005;294(9):1172 doi: doi:10.1001/jama.294.9.1172

5. Simon LV, Dove JC. When simulation becomes too real. Clin Teach 2018;15(3): 260-261. doi:10.1111/tct.12702

6. Marik PE, Varon, J. Hypertensive Crises: Challenges and Management. Chest. 2007;131(6):1949-1962. doi:10.1378/chest.06-2490

7. Goodenkauf, WA; Heesch, M; Hassenstab, B; Shute, RJ, Slivka, D (2015) "Acute High Intensity Anaerobic Training and Rhabdomyolysis Risk," International Journal of Exercise Science: Vol. 8 : Iss. 1

8. Knafl EG, Hughes JA, Dimeski G, Eley R. Rhabdomyolysis: Patterns, Circumstances, and Outcomes of Patients Presenting to the Emergency Department. Ochsner J. 2018;18(3):215-221. doi:10.31486/toj.17.0112

9. Olaiya MT, Hanson RL, Kavena KG, Sinha M, Clary D, Horton MB, Nelson RG, Knowler WC. Use of graded Semmes Weinstein monofilament testing for ascertaining peripheral neuropathy in people with and without diabetes. Diabetes Research and Clinical Practice. 2019;151:1-10. doi:10.1016/j.diabres.2019.03.029

10. Möltner A, Lehmann M, Wachter C, Kurczyk S, Schwill S, Loukanova S. Formative assessment of practical skills with peer-assessors: quality features of an OSCE in general medicine at the Heidelberg Medical Faculty. GMS J Med Educ. 2020 Jun 15;37(4):Doc42. doi: 10.3205/zma001335 
11. C. Puspitasari HP, Aslani P, Krass I. A review of counseling practices on prescription medicines in community pharmacies. Research in Social and Administrative Pharmacy. 2009;5(3)197-210. doi:10.1016/j.sapharm.2008.08.006

12. Tamblyn RM, Klass DJ, Schnable GK, Koppelow ML. The accuracy of standardised patient presentations. Med Educ 1991;25:100-9. doi:10.1016/S1607-551X(09)70031-2

13. Van der Vleuten CPM, Swanson DB. Assessment of clinical skills and standarised patients: state of the art. Teaching Learning Med 1990;2:58-76. doi:10.1080/10401339009539432

14. Al-Dahir S, Bryant K, Kennedy KB, Robinson DS. Online Virtual-Patient Cases Versus Traditional ProblemBased Learning in Advanced Pharmacy Practice Experiences. Am J Pharm Educ. 2014;78(4):76. doi:10.5688/ajpe78476

15. Shehata MHK, Abouzeid E, Wasfy NF, Abdelaziz A, Wells RL, Ahmed SA. Medical Education Adaptations Post COVID-19: An Egyptian Reflection. Journal of Medical Education and Curricular Development. 2020; (7). doi:10.1177/2382120520951819

16. Newble D, Dauphinee D, Dawson-Saunders B, et al. Guidelines for the development of effective and efficient procedures for the assessment of clinical competence. In: Newble DI, Jolly B, Wakeford R, eds. The certification and recertification of doctors: issues in the assessment of clinical competence. Cambridge: Cambridge University Press; 1994.

17. Cookson R, Dolan P, Principles of justice in health care rationing, Journal of Medical Ethics 2000;26:323-329. doi:10.1136/jme.26.5.323 